\title{
Reliability evaluation of modular multilevel converter based on Markov model
}

\author{
Liang ZHANG ${ }^{1,2}$ (D), Dan ZHANG ${ }^{3}$, Ting HUA ${ }^{1}$, Jihong ZHU $^{2}$, \\ Gang CHEN ${ }^{4}$, Tongzhen $\mathrm{WEI}^{5}$, Ting YANG ${ }^{1}$
}

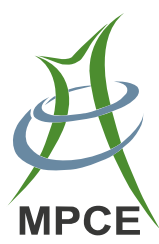

\begin{abstract}
The modular multilevel converter (MMC) is now the most attractive topology for medium and high voltage power conversion applications with several advantages over the traditional voltage source converter (VSC). However, due to a large number of sub-modules (SMs) in the MMC, system reliability is a big challenge in its practical application, where each SM may be considered as a potential point of failure. In this paper, a reliability evaluation based on the Markov model is proposed for the MMC. The failure rates of the power electronic devices and SMs are firstly analyzed. Then, the Markov model and the state transition equation of the system are built in detail. A general reliability evaluation function is established, in which the mean time to failure and reliability evaluation of the MMC with redundant SMs are also discussed. Finally, a practical direct current (DC) distribution example for reliability evaluation is analyzed, and the results verify that
\end{abstract}

CrossCheck date: 21 December 2018

Received: 23 December 2017 / Accepted: 24 December 2018/
Published online: 3 April 2019
@ The Author(s) 2019
\Liang ZHANG
zhldream@126.com
Dan ZHANG
910111564@qq.com
Ting HUA
444155792@qq.com
Jihong ZHU
jhzhu@tsinghua.edu.cn
Gang CHEN
njgd2001@aliyun.com
Tongzhen WEI
tzwei@mail.iee.ac.cn

the reliability evaluation based on the Markov model could provide a useful reference for project design.

Keywords Modular multilevel converters, Reliability evaluation, Markov model

\section{Introduction}

The offshore wind power has been widely developed in Europe, China, the United States and Australia [1]. Voltage source converter (VSC) based high voltage direct current (HVDC) transmission systems, including multi-terminal HVDC systems, are widely applied to transmit the power $[2,3]$, in which the modular multilevel converter (MMC) topology attracts the most attention of researchers [4]. The MMC topology is also used in DC distribution and power quality control, such as a unified power flow controller (UPFC) and static synchronous compensator (STATCOM) $[5,6]$. Most research has focused on the operation and control

Ting YANG

279730157@qq.com

1 School of Power Electric Engineering, Nanjing Institute of Technology, Nanjing 211167, China

2 State Key Laboratory of Intelligent Technology and Systems, Tsinghua University, Beijing 100084, China

3 School of Electronic Information and Electrical Engineering, Shanghai Jiao Tong University, Shanghai 200240, China

4 State Grid Jiangsu Electric Power Company Xuzhou Power Supply Company, Xuzhou 221005, China

5 Institute of Electrical Engineering, Chinese Academy of Sciences, Beijing 100190, China 
strategy of MMCs [7-11], while their failure prediction has not received much discussion, although it is an important issue in power systems $[12,13]$. Hence, there is a big motivation to find a solution for reliability evaluation and submodule (SM) redundancy configuration of the MMC.

Some tentative work has been done in previous studies. For example, redundancy and reliability indexes of the MMC were defined in [14], but the general reliability function was not built. Also, using the k-out-of-n: G model and Gamma distribution, a reliability function of MMC was established [15], wherein the failure rates of SM components were based on the experience-hypothesis. Explicit models of components were not given. Some research has focused on the optimal number of SMs, without considering reliability evaluation $[16,17]$.

In this paper, the Markov model is proposed to analyze the failure rates of the MMC and its SMs, based on a graphical representation of system states. A general function for reliability evaluation of the MMC is given. The mean time to failure and reliability evaluation of the MMC with a redundant SM are both discussed.

The paper is organized as follows. In Section 2, the basic operation principles of the MMC are presented. In Section 3, the cause and impact of SM failure are discussed. Reliability analysis of MMC is given in Section 4, and with a redundant SM in Sections 5. Finally, an example analysis is provided in Section 6.

\section{Basic operation principles of MMC}

A three-phase MMC is shown in Fig. 1, which consists of six arms, with $n$ SMs in each arm [18]. $L_{0}$ is the arm inductor; $U_{d c}$ is the DC bus voltage; $u_{j m}(j=\mathrm{a}, \mathrm{b}, \mathrm{c})$ is the output voltage of phase $j$ and $u_{c}$ is the capacitor rated voltage.

Each SM has three states, as shown in Table 1. State A is called "ON" state, where switch $S_{1}$ is ON and switch $S_{2}$ is OFF. The output voltage is $u_{c}$. State B is called the "OFF" state, where switch $S_{1}$ is OFF switch $S_{2}$ is ON. The output voltage is $0 \mathrm{~V}$. State $\mathrm{C}$ is called the "BLOCK" state, where switch $S_{1}$ and switch $S_{2}$ are OFF. In the "BLOCK" state, when the current is flowing into the $\mathrm{SM}, i_{S M}$ is positive and the output voltage is $u_{c}$. When the current is flowing out of $\mathrm{SM}, i_{S M}$ is negative and the output voltage is $0 \mathrm{~V}$.

\section{Cause and impact of SM fault}

Each SM may be considered as a potential point of failure [19]. Hence, it is important to know the causes and impact of a SM fault.

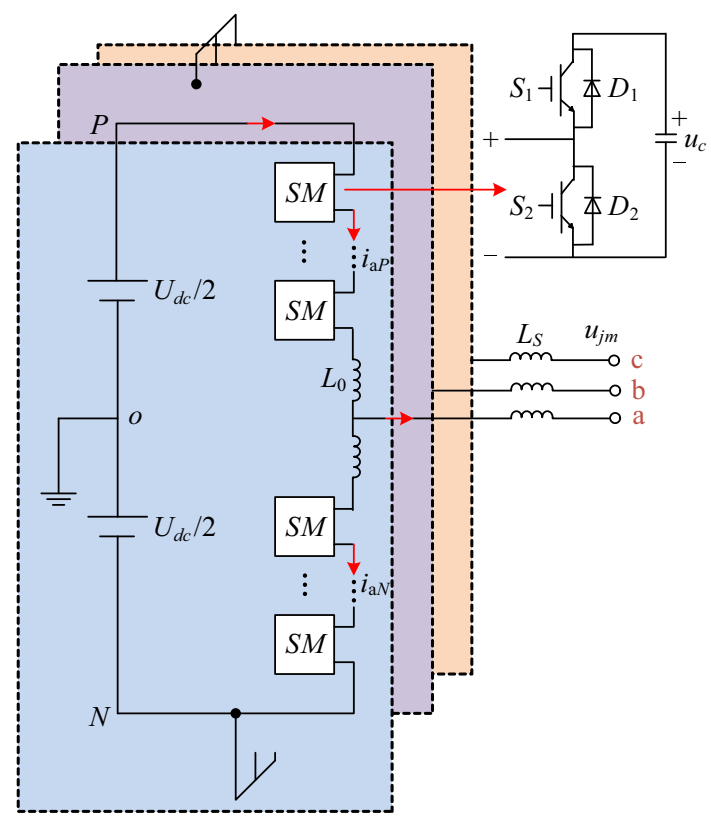

Fig. 1 Diagram of MMC

Table 1 Working mode of SM

\begin{tabular}{lllll}
\hline State & $S_{1}$ & $S_{2}$ & $i_{S M}$ & $u_{S M}$ \\
\hline ON & ON & OFF & & $u_{c}$ \\
OFF & OFF & ON & & 0 \\
BLOCK & OFF & OFF & Positive & $u_{c}$ \\
& & & Negative & 0 \\
\hline
\end{tabular}

\subsection{Causes of SM fault}

There are three main causes to trigger the failure of a SM.

1) Damage to a power electronic component

The overload capability of power electronic components, such as insulated gate bipolar transistors (IGBTs) and diodes, is limited, so overvoltage and overcurrent might break them. Therefore, the damage to a power electronic component is one of the most common reasons to cause the SM fault.

2) Damage to a passive component

The DC capacitor is a key passive component in a SM, and damage to it would also cause a SM fault. Fortunately, its failure rate is lower than that of power electronic devices. 
3) A faulty trigger pulse

If the trigger pulse signal experiences interference, it could not operate the SM correctly, and this might lead the SM to a fault in either short circuit or open circuit mode [20].

\subsection{Impacts of SM fault}

Once one SM is damaged, it would be bypassed and then replaced by the redundant SM, which can cause distortion of the output voltage and current and reduced performance of the converter. A fault of one SM can also cause the other components in the corresponding arm to fail and lead to total system collapse. When the quantity of broken SMs is more than the redundant ones, the MMC would work with asymmetrical operation. In the worst case, this might result in system failure.

\section{Reliability analysis of MMC}

To evaluate the MMC's reliability, an analysis of the reliability of semiconductor devices (IGBTs and diodes) and capacitors is first required for use in the Markov model.

\subsection{Component failure rate}

Power electronic components play an important role in reliability of the whole system, so it is necessary to estimate their failure rates [21, 22]. An extensive database of various types of parts is provided in the RDF-2000 [23], which is widely accepted and frequently used to determine the reliability of electronic equipment. Using these data, mathematical models for power IGBT, aluminum electrolytic capacitor and power diode failure rates are expressed in (1), (2) and (3) respectively. The unit of failure rates is $10^{-9} / \mathrm{h}$.

$$
\begin{aligned}
\lambda_{I G B T}= & (\underbrace{\left(\pi_{S} \lambda_{0}\right) \frac{\sum_{i=1}^{y}\left(\pi_{t}\right)_{i} \tau_{i}}{\tau_{\text {on }}+\tau_{\text {off }}}}_{\lambda_{\text {die }}}+\underbrace{\pi_{I} \lambda_{\text {EOS }}}_{\lambda_{\text {over }}} \\
& +\underbrace{\left(2.75 \times 10^{-3} \sum_{i=1}^{z}\left(\pi_{n}\right)_{i}\left(\Delta T_{i}\right)^{0.68}\right) \lambda_{B}}_{\lambda_{\text {pack }}})
\end{aligned}
$$

$$
\begin{aligned}
\lambda_{\text {diode }}= & (\underbrace{\left(\pi_{U} \lambda_{0}\right) \frac{\sum_{i=1}^{y}\left(\pi_{t}\right)_{i} \tau_{i}}{\tau_{\text {on }}+\tau_{\text {off }}}}_{\lambda_{\text {die }}}+\underbrace{\pi_{I} \lambda_{E O S}}_{\lambda_{\text {over }}} \\
+ & \underbrace{\left(2.75 \times 10^{-3} \sum_{i=1}^{z}\left(\pi_{n}\right)_{i}\left(\Delta T_{i}\right)^{0.68}\right) \lambda_{B}}_{\lambda_{\text {pack }}}) \\
\lambda_{\text {cap }}= & 2.4 \times\left(\frac{\sum_{i=1}^{y}\left(\pi_{t}\right)_{i} \tau_{i}}{\tau_{\text {on }}+\tau_{\text {off }}}+1.4 \times 10^{-3} \sum_{i=1}^{z}\left(\pi_{n}\right)_{i}\left(\Delta T_{i}\right)^{0.68}\right)
\end{aligned}
$$

where $\lambda_{I G B T}$ is the failure rate of the IGBT; $\lambda_{\text {diode }}$ is the failure rate of the diode; $\lambda_{\text {cap }}$ is the failure rate of the capacitor; $\lambda_{0}$ is the base failure rate of the die; $\pi_{S}$ is the charge factor; $\left(\pi_{t}\right)_{i}$ is the $i^{\text {th }}$ temperature factor related to the $i^{\text {th }}$ junction temperature of the transistor mission profile; $\tau_{i}$ is the $i^{\text {th }}$ working time ratio for the $i^{\text {th }}$ junction temperature of the mission profile; $\tau_{o n}$ is the total working time ratio; $\tau_{\text {off }}$ is the time ratio in storage (or dormant) mode; $\lambda_{\text {die }}$ is the failure rate of the die; $\pi_{I}$ is the influence factor related to usage (protection interface or not); $\lambda_{E O S}$ is the failure rate related to the electrical overstress in the application being considered; $\lambda_{\text {over }}$ is the failure rate of the overstress; $\left(\pi_{n}\right)_{i}$ is the $i^{\text {th }}$ influence factor related to the annual cycles of thermal variation seen by the package, with the amplitude $\Delta T_{i} ; \Delta T_{i}$ is the $i^{\text {th }}$ thermal amplitude variation of the mission profile; $\lambda_{B}$ is the base failure rate of the package; $\lambda_{\text {pack }}$ is the failure rate of the package; $\pi_{U}$ is the use factor.

According to the IGBT model and the rated working conditions of a practical system in Section 6, the average dissipated power of diode and IGBT is calculated as follows:

1) The average power dissipated by the diode is $53.47 \mathrm{~W}$.

2) The average power dissipated by IGBT is $346 \mathrm{~W}$.

3) The junction-ambient thermal resistance is $0.1618{ }^{\circ} \mathrm{C} /$ W.

Some corresponding fault parameter values of components are given in Table 2. Based on the failure rate models of components, the failure rates of IGBTs, diodes, and capacitors in the MMC have been calculated as shown.

\subsection{Summary of Markov reliability model}

The Markov model can be used to estimate a variety of reliability indexes, such as the failure rate, the mean time to 
Table 2 Parameters for failure rates of components

\begin{tabular}{llll}
\hline Parameter & IGBT & Capacitor & Diode \\
\hline$\lambda_{0}$ & 2 & & 0.7 \\
$\pi_{U}$ & & & 1 \\
$\pi_{S}$ & 0.48 & & \\
$\lambda_{B}$ & 10 & & 10 \\
$\pi_{I}$ & 1 & & 1 \\
$\lambda_{\text {EOS }}$ & 40 & 0.942 & 40 \\
$\tau_{\text {off }}$ & 0.942 & 0.058 & 0.942 \\
$\tau_{\text {on }}$ & 0.058 & 18.33 & 0.058 \\
Failure rate & 224.535 & & 194.32 \\
\hline
\end{tabular}

Note: The unit of failure rates is $10^{-9} / \mathrm{h}$

failure (MTTF), the reliability and the availability $[24,25]$.

First of all, a random state function $\{X(t), t>0\}$ is defined. At time $t$, the probability of the system in the $i^{\text {th }}$ state can be expressed as:

$P_{i}(t)=P\{X(t)=i\}$

With the transition to the $j^{\text {th }}$ state after time $\Delta t$, the probability $P_{i j}$ can be expressed as:

$P_{i j}(t)=P\{X(t+\Delta t)=j \mid X(t)=i\}$

The transition rate $\lambda_{i j}$ that denotes the probability of the system transiting from the $i^{\text {th }}$ state to the $j^{\text {th }}$ state at time $t$ is:

$\lambda_{i j}=\lim _{\Delta t \rightarrow 0} \frac{P_{i j}(t)}{\Delta t}$

The system transitions between different states due to faults and maintenance of the system components. The three-state transition diagram [26-28] is shown in Fig. 2. $\mu_{10}$ is the returning rate; State 0 stands for the system works well; State 1 stands for the system has a fault, but could still work; State 2 stands for the system could no longer work.

The state equation can be expressed as:

$\frac{\mathrm{d}}{\mathrm{d} t}\left[\begin{array}{l}P_{0} \\ P_{1} \\ P_{2}\end{array}\right]=\left[\begin{array}{ccc}-\left(\lambda_{01}+\lambda_{02}\right) & \mu_{10} & 0 \\ \lambda_{01} & -\left(\mu_{10}+\lambda_{12}\right) & 0 \\ \lambda_{02} & \lambda_{12} & 0\end{array}\right]\left[\begin{array}{l}P_{0} \\ P_{1} \\ P_{2}\end{array}\right]$

The probabilities of the system can be obtained by solving the state equation (7). $P_{0}, P_{1}$ and $P_{2}$ are the probabilities of the system being in state 0 , state 1 and state 2 , respectively. $P_{2}$ is also called the failure rate. The reliability function of the system is the sum of probability functions of all functional (non-failed) states, which is mathematically expressed as:

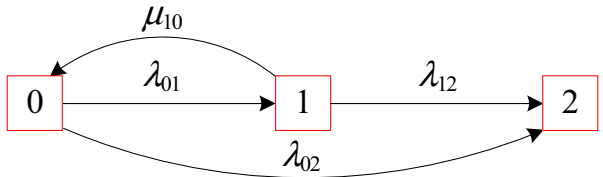

Fig. 2 State transition diagram

$R(t)=P_{0}+P_{1}$

Then, the MTTF can be derived as:

$M T T F=\int_{0}^{\infty} R(t) \mathrm{d} t$

\subsection{Markov model of MMC reliability evaluation without redundant SMs}

To reduce the order of the state equation and simplify the model, the same operating states and transition processes are assumed to apply to the SM. The reliability of the system could be considered as a summary of the reliability of multiple SMs. When the SM exits a fault state, it is considered as a permanent fault. Hence, the rate of return is equal to 0 .

The Markov model of the MMC is shown in Fig. 3.

where State 0 is the normal working state, and State 1 is the abnormal state, which means that there is one SM fault in any arm. The failure rate of SMs can be obtained by calculating the failure rates of the IGBTs, capacitors, and diodes in it.

Using the data in Table 2, the failure rate of a SM can be expressed in (10), because there are two IGBTs, two diodes, and one capacitor in each SM:

$\lambda_{S M}=2 \lambda_{I G B T}+2 \lambda_{\text {diode }}+\lambda_{\text {cap }}=856 \times 10^{-9} / \mathrm{h}$

where $\lambda_{S M}$ is the failure rate of the SM.

With $6 n$ SMs in the MMC, $\lambda_{01}$ can be obtained as:

$\lambda_{01}=6 n \lambda_{S M}=5.14 \times 10^{-6} n$

Therefore equation (7) can be rewritten as:

$\frac{\mathrm{d}}{\mathrm{d} t}\left[\begin{array}{l}P_{0} \\ P_{1}\end{array}\right]=\left[\begin{array}{cc}-5.136 \times 10^{-6} n & 0 \\ 5.136 \times 10^{-6} n & 0\end{array}\right]\left[\begin{array}{l}P_{0} \\ P_{1}\end{array}\right]$

with initial conditions: $P_{0}(0)=1 ; P_{1}(0)=0$.

Solving (12) determines that:

$\left\{\begin{array}{l}P_{0}(t)=\mathrm{e}^{-5.136 \times 10^{-6} n t} \\ P_{1}(t)=1-\mathrm{e}^{-5.136 \times 10^{-6} n t}\end{array}\right.$

Therefore, the reliability function of the MMC is:

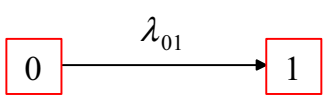

Fig. 3 Markov model of MMC 


$$
R(t)=\mathrm{e}^{-5.136 \times 10^{-6} n t}
$$

the failure rate function of the MMC is:

$P(t)=1-\mathrm{e}^{-5.136 \times 10^{-6} n t}$

and the MTTF function is:

$M T T F=\int_{0}^{+\infty} \mathrm{e}^{-5.136 \times 10^{-6} n t} \mathrm{~d} t=\frac{1.947 \times 10^{5}}{n}$

where $n$ is the number of SMs in any arm. Obviously, with an increasing number of SMs, the MTTF reduces proportionally.

The changing reliability and failure rate of the system with time and the number of SMs per arm $(n)$ are shown respectively in Fig. 4 and Fig. 5.

One can see that with fewer than 50 modules, the reliability does not drop rapidly with time, and before 1000 hours of operation, the reliability does not fall quickly with the number of SMs.

\section{Reliability evaluation of MMC with a redundant SM by Markov model}

As above, a general function for the reliability evaluation of the basic MMC is obtained.

Firstly, there are three main modes for the redundant operation of the SM [29, 30]:

1) The redundant SMs do not participate in normal operation of the MMC. When a SM fails, it is removed, and the redundant SM is put into operation.

2) The redundant SMs participate in normal operation. When a SM fails, it is removed, and the corresponding normal SM in the other phase is bypassed so that the system operates symmetrically.

3) The redundant SMs participate in normal operation. When a SM fails, only that SM is bypassed.

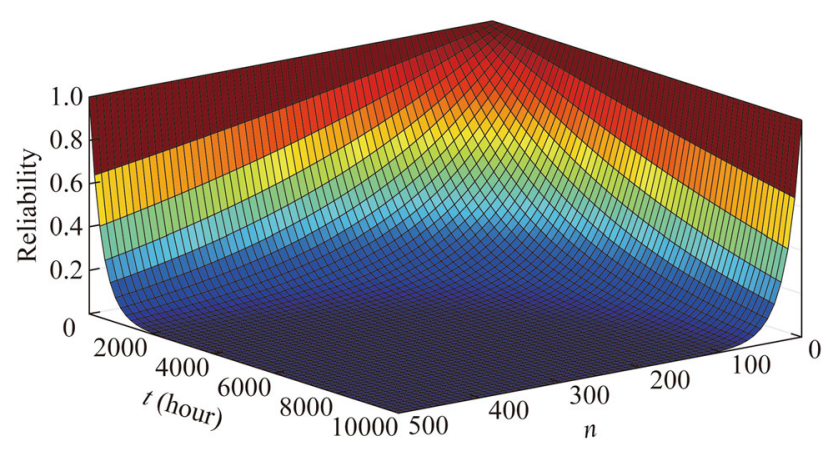

Fig. 4 Reliability function of MMC

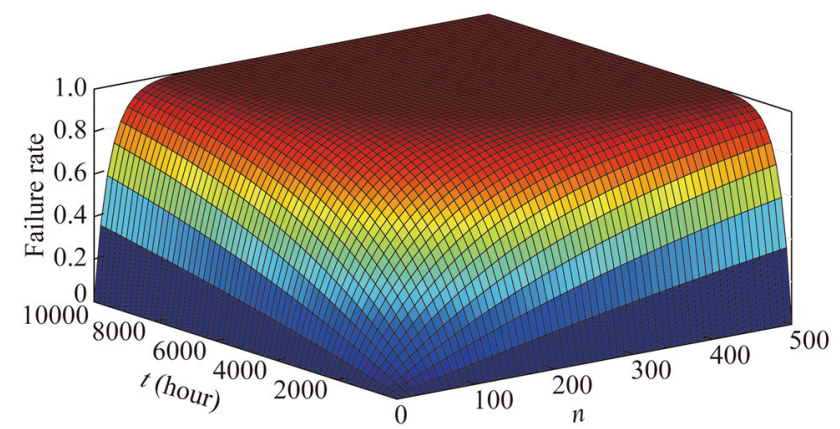

Fig. 5 Failure rate function of MMC

Mode 3 leads to asymmetric operation. Mode 2 is not economical because the corresponding SM in the other phase is removed and the redundant SMs are aged by operation. Therefore, mode 1 is chosen for discussion.

The Markov model for mode 1 is shown in Fig. 6, where the numbers of SMs and cold standby redundant SMs $\left(n_{0}\right)$ in each arm are $n$ and one, respectively.

There are eight states in the model. State 0: All devices work well; State 1: One SM in any arm fails; State 2: Any two arms both have one SM failing; State 3, 4, 5: Respectively any three, four, five arms all have one SM failing; State 6: Six bridge arms all have one SM failing; State 7: The system can no longer work.

Take the transition from State 1 to State 2 as an example. Supposing that the MMC is in state 1, and an SM on the upper arm of phase A fails. Then, the faulty SM is removed and the redundant SM is put to use. The diagram of MMC in State 1 is shown in Fig. 7.

If another SM fails in one of the other five arms, the MMC can still run. The state transfers from state 1 to state 2. If another SM fails in the same arm, the MMC cannot run. The state transfers from state 1 to state 7 .

Therefore, $\lambda_{12}$ and $\lambda_{17}$ are determined by:

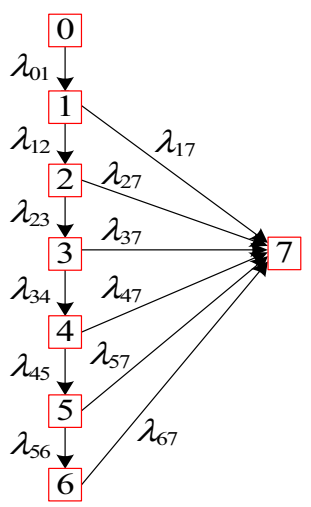

Fig. 6 Markov model of MMC with a redundant SM 
$\left\{\begin{array}{l}\lambda_{12}=5 \lambda \\ \lambda_{17}=\lambda\end{array}\right.$

where $\lambda$ is the failure rate of any arm and could be expressed as:

$\lambda=856 n \times 10^{-9} / \mathrm{h}$

Other state transitions can be derived in a similar way, and the transition rates are:

$\left\{\begin{array}{l}\lambda_{01}=\lambda_{67}=6 \lambda \\ \lambda_{23}=\lambda_{47}=4 \lambda \\ \lambda_{57}=5 \lambda \\ \lambda_{34}=\lambda_{37}=3 \lambda \\ \lambda_{45}=\lambda_{27}=2 \lambda \\ \lambda_{56}=\lambda\end{array}\right.$

The equation of state can be expressed as:

$\mathrm{d}\left[\begin{array}{c}P_{0} \\ P_{1} \\ P_{2} \\ P_{3} \\ P_{4} \\ P_{5} \\ P_{6} \\ P_{7}\end{array}\right]=\left[\begin{array}{cccccccc}-6 \lambda & 0 & 0 & 0 & 0 & 0 & 0 & 0 \\ 6 \lambda & -6 \lambda & 0 & 0 & 0 & 0 & 0 & 0 \\ 0 & 5 \lambda & -6 \lambda & 0 & 0 & 0 & 0 & 0 \\ 0 & 0 & 4 \lambda & -6 \lambda & 0 & 0 & 0 & 0 \\ 0 & 0 & 0 & 3 \lambda & -6 \lambda & 0 & 0 & 0 \\ 0 & 0 & 0 & 0 & 2 \lambda & -6 \lambda & 0 & 0 \\ 0 & 0 & 0 & 0 & 0 & \lambda & -6 \lambda & 0 \\ 0 & \lambda & 2 \lambda & 3 \lambda & 4 \lambda & 5 \lambda & 6 \lambda & 0\end{array}\right]\left[\begin{array}{c}P_{0} \\ P_{1} \\ P_{2} \\ P_{3} \\ P_{4} \\ P_{5} \\ P_{6} \\ P_{7}\end{array}\right]$

The sum of all states' probabilities is:

$\sum_{i=0}^{7} P_{i}(t)=1$

The initial conditions are $P_{0}(0)=1$; $P_{i}(0)=0(i=1 \sim 7)$.

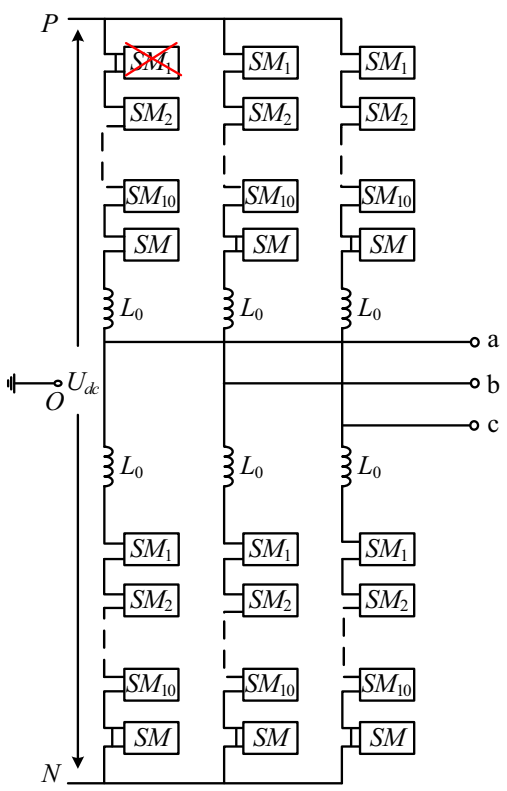

By solving (20) subject to (21) these probability functions are obtained:

$$
\left\{\begin{aligned}
P_{0}(t) & =\mathrm{e}^{-6 \lambda t} \\
P_{1}(t) & =6 \lambda t \mathrm{e}^{-6 \lambda t} \\
P_{2}(t) & =15 \lambda^{2} t^{2} \mathrm{e}^{-6 \lambda t} \\
P_{3}(t) & =20 \lambda^{3} t^{3} \mathrm{e}^{-6 \lambda t} \\
P_{4}(t) & =15 \lambda^{4} t^{4} \mathrm{e}^{-6 \lambda t} \\
P_{5}(t) & =6 \lambda^{5} t^{5} \mathrm{e}^{-6 \lambda t} \\
P_{6}(t) & =\lambda^{6} t^{6} \mathrm{e}^{-6 \lambda t} \\
P_{7}(t) & =1-\lambda^{6} t^{6} \mathrm{e}^{-6 \lambda t}-6 \lambda^{5} t^{5} \mathrm{e}^{-6 \lambda t}-15 \lambda^{4} t^{4} \mathrm{e}^{-6 \lambda t} \\
& -20 \lambda^{3} t^{3} \mathrm{e}^{-6 \lambda t}-15 \lambda^{2} t^{2} \mathrm{e}^{-6 \lambda t}-6 \lambda t \mathrm{e}^{-6 \lambda t}-\mathrm{e}^{-6 \lambda t}
\end{aligned}\right.
$$

The reliability function is therefore:

$$
\begin{aligned}
R(t) & =\sum_{i=0}^{6} P_{i} \\
& =\lambda^{6} t^{6} \mathrm{e}^{-6 \lambda t}+6 \lambda^{5} t^{5} \mathrm{e}^{-6 \lambda t}+15 \lambda^{4} t^{4} \mathrm{e}^{-6 \lambda t} \\
& +20 \lambda^{3} t^{3} \mathrm{e}^{-6 \lambda t}+15 \lambda^{2} t^{2} \mathrm{e}^{-6 \lambda t}+6 \lambda t \mathrm{e}^{-6 \lambda t}+\mathrm{e}^{-6 \lambda t}
\end{aligned}
$$

and the MTTF function with a redundant SM is:

$$
\begin{aligned}
\text { MTTF } & =\int_{0}^{+\infty}\left(\lambda^{6} t^{6} \mathrm{e}^{-6 \lambda t}+6 \lambda^{5} t^{5} \mathrm{e}^{-6 \lambda t}+15 \lambda^{4} t^{4} \mathrm{e}^{-6 \lambda t}\right. \\
& \left.+20 \lambda^{3} t^{3} \mathrm{e}^{-6 \lambda t}+15 \lambda^{2} t^{2} \mathrm{e}^{-6 \lambda t}+6 \lambda t \mathrm{e}^{-6 \lambda t}+\mathrm{e}^{-6 \lambda t}\right) \mathrm{d} t \\
& =\frac{7.349 \times 10^{5}}{n}
\end{aligned}
$$

The changing reliability of this MMC with time and the number of SMs per arm (n) is shown in Fig. 8.

The work above could provide a theoretical basis for a practical application project. By using Markov model, a general function and a mathematical calculation is firstly presented for the reliability of MMC, although it is always difficult to give a precise reliability evaluation for MMC in a practical power system project, with a large amount of power electronic components.

\section{A reliability evaluation for a practical distribution network project}

In this section, a reliability evaluation of MMC is given for a practical DC distribution network project in Zhejiang Province, China. Some parameter values of MMC are shown in Table 3.

The structure diagram of the DC distribution system in this project is shown in Fig. 9, where the rated DC voltage is $10 \mathrm{kV}$ and the rated power is $10 \mathrm{MW}$. Each arm consists of 24 SMs.

Fig. 7 Diagram of MMC in state 1 


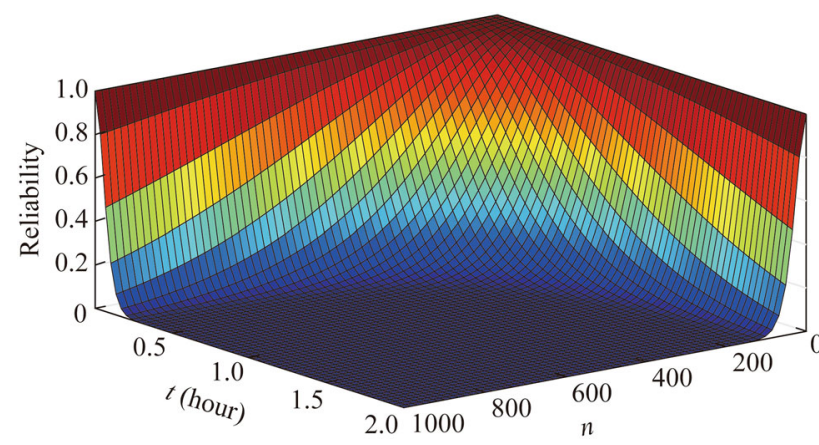

Fig. 8 Reliability function of the MMC with a redundant SM

Table 3 Some parameter values of the MMC used in a practical project in Zhejiang

\begin{tabular}{ll}
\hline Parameter & Value \\
\hline Rated DC voltage of SM & $900 \mathrm{~V}$ \\
Rated current of SM & $289 \mathrm{~A}$ \\
Switching frequency & $1100 \mathrm{~Hz}$ \\
Modulation ratio & 0.95 \\
Power factor & 0.99 \\
\hline
\end{tabular}

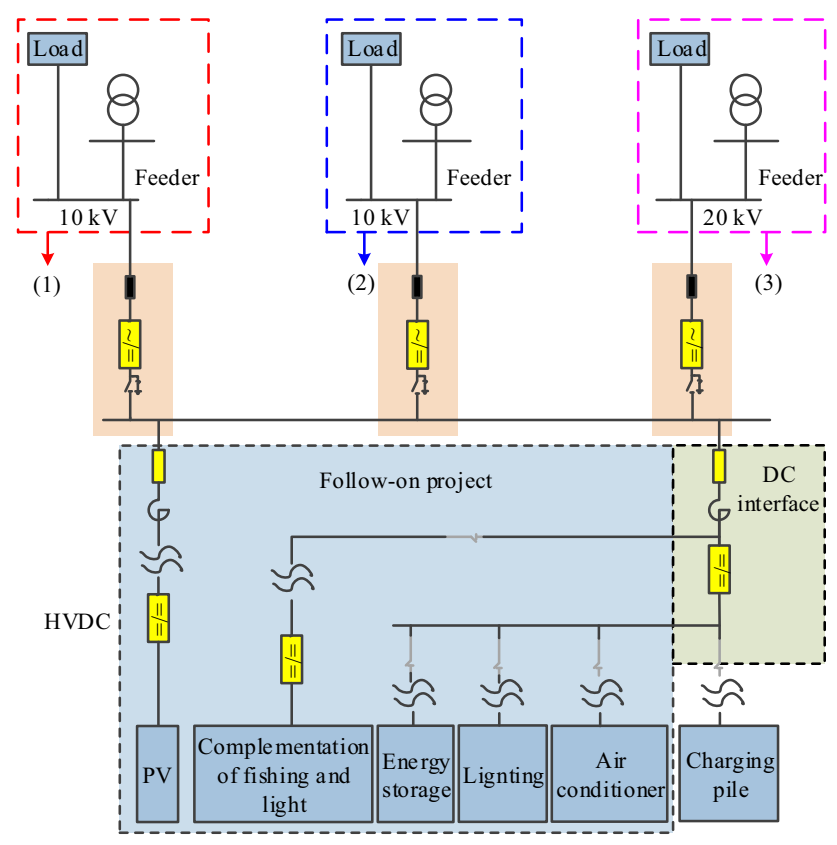

(1): Transformer substation of Linxin, China

(2): Transformer substation of Long March, China

(3): Transformer substation of New Bay, China

$\rightarrow-$ AC circuit breaker; $\square-$ DC circuit breaker $\sim=$ HVDC; $=/=$ DC transformer

Fig. 9 Structure diagram of a practical DC distribution system project in Zhejiang

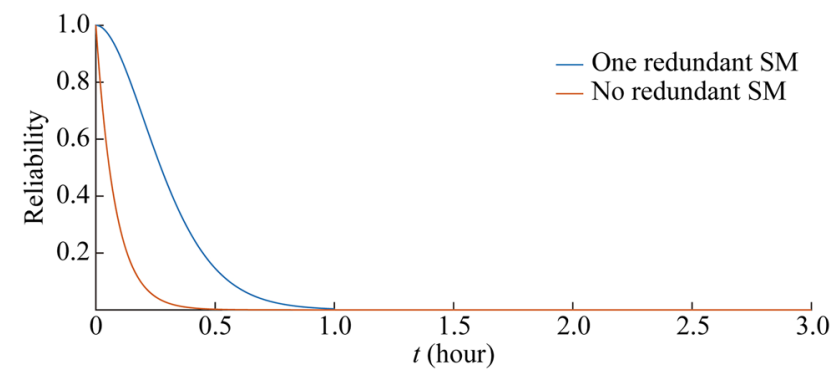

Fig. 10 Comparison of the reliability with and without a redundant SM

Table 4 Comparison of two MTTFs

\begin{tabular}{lc}
\hline Case & MTTF (days) \\
\hline Without redundant SM & $<400$ \\
One redundant SM & $>1200$ \\
\hline
\end{tabular}

By the project parameters above and the evaluation model, it could be found that the failure rate of any arm is $2.054 \times 10^{-5} / \mathrm{h}$. Furthermore, according to (14) and (23) the reliability of the project converter with a redundant SM $R_{1}(t)$ and without a redundant SM $R_{2}(t)$ could be derived as follows

$$
\left\{\begin{aligned}
R_{1}(t) & =\mathrm{e}^{-1.23 \times 10^{-4} t} \\
R_{2}(t) & =\left[\left(2.054 \times 10^{-5}\right)^{6} t^{6}+6\left(2.054 \times 10^{-5}\right)^{5} t^{5}\right. \\
& +15\left(2.054 \times 10^{-5}\right)^{4} t^{4}+20\left(2.054 \times 10^{-5}\right)^{3} t^{3} \\
& \left.+15\left(2.054 \times 10^{-5}\right)^{2} t^{2}+6\left(2.054 \times 10^{-5}\right) t+1\right] \mathrm{e}^{-1.23 \times 10^{-4} t}
\end{aligned}\right.
$$

They are compared in Fig. 10.

Based on (16) and (24), the MTTF of the system with and without a redundant SM is more than 1200 days and less than 400 days respectively, as shown in Table 4.

The evaluation method proposed has a distinct effect to give a theoretical analysis and reliability evaluation for a practical project. For the DC distribution network project in Zhejiang, it could be found that the MTTF of the system with a redundant $\mathrm{SM}$ is more than 3 times that of the system without a redundant SM. Hence, it provides a theoretical proof that there should be at least one redundant SM in each arm to obtain a high reliability.

\section{Conclusion}

In a practical power system project, it is always difficult to provide a precise reliability evaluation for MMC because there is a large amount of power electronic components. In this paper, the Markov model is proposed to 
evaluate the reliability of the MMC. Based on the model, the mathematical calculation and a general function are firstly presented considering the failure of the electronic equipment in MMC. Based on that, the mean time to failure and the influence of redundant SMs are both discussed. Finally, they are applied to reliability evaluation for a practical DC distribution system. The system with a single redundant SM provides a factor of more than 3 improvement in reliability. The results give a further validation that the reliability evaluation based on Markov model could provide a useful reference for practical project design.

Acknowledgements This work was supported by the National Natural Science Foundation of China (No. 51607084), the National Key Research and Development Program of China (No. 2017YFB0903504), Jiangsu Electric Power Company Project (No. J2018076), State Grid Technology Project (No. 5210EF17002B) and the State Key Laboratory of Smart Grid Protection and Control.

Open Access This article is distributed under the terms of the Creative Commons Attribution 4.0 International License (http:// creativecommons.org/licenses/by/4.0/), which permits unrestricted use, distribution, and reproduction in any medium, provided you give appropriate credit to the original author(s) and the source, provide a link to the Creative Commons license, and indicate if changes were made.

\section{References}

[1] Chi Y, Liang W, Zhang Z et al (2016) An overview on key technologies regarding power transmission and grid integration of large-scale offshore wind power. Proc CSEE 36(14):3758-3770

[2] Cheah-Mane M, Sainz L, Liang J et al (2017) Criterion for the electrical resonance stability of offshore wind power plants connected through HVDC links. IEEE Trans Power Syst 32(6):4579-4589

[3] Adam P, Gowaid A, Finney J et al (2016) Review of DC-DC converters for multi-terminal HVDC transmission networks. IET Power Electron 9(2):281-296

[4] Hahn F, Andresen M, Buticchi G et al (2018) Thermal analysis and balancing for modular multilevel converters in HVDC applications. IEEE Trans Power Syst 33(3):1985-1996

[5] Kontos E, Tsolaridis G, Teodorescu R et al (2017) High order voltage and current harmonic mitigation using the modular multilevel converter STATCOM. IEEE Access 5:16684-16692

[6] Chen G, Li P, Yuan Y (2016) Application of MMC-UPFC on Nanjing Western Grid and its harmonic analysis. Autom Electr Power Syst 40(7):121-127

[7] Basante A, Ceballos S, Konstantinou G et al $(2018)(2 N+1)$ selective harmonic elimination-PWM for modular multilevel converters: a generalized formulation and a circulating current control method. IEEE Trans Power Electron 33(1):802-817

[8] Dekka A, Wu B, Fuentes R et al (2017) Evolution of topologies, modeling, control schemes, and applications of modular multilevel converters. IEEE J Emerg Select Topics Power Electron 5(4):1631-1656

[9] Debnath S, Qin J, Bahrani B et al (2015) Operation, control, and applications of the modular multilevel converter: a review. IEEE Trans Power Electron 30(1):37-53
[10] Beza M, Bongiorno M, Stamatiou G (2018) Analytical derivation of the AC-side input admittance of a modular multilevel converter with open- and closed-loop control strategies. IEEE Trans Power Deliv 33(1):248-256

[11] Perez A, Rodriguez J, Kouro S (2015) Circuit topologies, modeling, control schemes, and applications of modular multilevel converters. IEEE Trans Power Electron 30(1):4-17

[12] Guan M, Xu Z (2011) Redundancy protection for sub-module faults in modular multilevel converter. Autom Electr Power Syst 35(16):94-104

[13] Høyland A, Rausand M (1994) System reliability theory: models and statistical methods. Wiley, New York

[14] Wang C, Zhao C, Xu J (2013) A method for calculating submodule redundancy configurations in modular multilevel converters. Autom Electr Power Syst 37(16):103-107

[15] Wang X, Guo J, Pang H (2016) Structural reliability analysis of modular multi-level converters. Proc CSEE 36(7):1908-1914

[16] Xu J, Zhao P, Zhao C (2016) Reliability analysis and redundancy configuration of MMC with hybrid submodule topologies. IEEE Trans Power Electron 31(4):2720-2729

[17] Wang B, Tan F, Shang J (2015) Optimal configuration of modular redundancy for MMC. Electric Power Autom Equip 35(1):13-19

[18] Peng H, Deng Y, Wang Y (2015) Research about the model and steady-state performance for modular multilevel converter. Trans China Electrotech Soc 30(12):37-53

[19] Richardeau F, Pham T (2013) Reliability calculation of multilevel converters: theory and applications. IEEE Trans Ind Electron 60(10):4225-4233

[20] Ghazanfari A, Abdel-Rady Y (2016) A resilient framework for fault-tolerant operation of modular multilevel converters. IEEE Trans Ind Electron 63(5):2669-2678

[21] Gadalla B, Schaltz E, Blaabjerg F (2015) A survey on the reliability of power electronics in electro-mobility applications. In: Proceedings of 2015 international Aegean conference on electrical machines \& power electronics (ACEMP), 2015 International conference on optimization of electrical \& electronic equipment (OPTIM) \& 2015 International symposium on advanced electromechanical motion systems (ELECTROMOTION), Side, Turkey, 2-4 September 2015, pp 304-310

[22] Song Y, Wang B (2012) A hybrid electric vehicle powertrain with fault- tolerant capability. In: Proceedings of 2012 twentyseventh annual IEEE applied power electronics conference \& exposition, Orlando, USA, 5-9 February 2012, pp 951-956

[23] PD IEC TR 62380:2004. Reliability data handbook-universal model for reliability prediction of electronics components, PCBs and equipment. https://shop.bsigroup.com/ProductDetail/?pid= 000000000030096545. Accessed 8 November 2004

[24] Brinzei N, Nahid-Mobarakeh B (2014) Reliability assessment of adjustable speed drives using state Markov models. In: Proceedings of 2014 IEEE industry application society annual meeting, Vancouver, Canada, 5-9 October 2014, pp 1-8

[25] Najmi V, Wang J, Burgos R et al (2015) Reliability modeling of capacitor bank for modular multilevel converter based on Markov state-space model. In: Proceedings of 2015 IEEE applied power electronics conference and exposition (APEC), Charlotte, USA, 15-19 March 2015, pp 2703-2709

[26] Pukite J, Pukite P (1998) Modeling for reliability analysis: Markov modeling for reliability, maintainability, safety, and supportability analyses of complex systems. Wiley-IEEE Press, New York

[27] Butler RW, Johnson SC (1995) Techniques for modeling the reliability of fault-tolerant systems with the Markov state-space approach. NASA Reference Publication, 1995

[28] Garg D, Makkar P (2015) Markov modelling for reliability analysis. Int J Appl Eng Res 10(35):27547-27552 
[29] Li B, Zhang Y, Yang R et al (2015) Seamless transition control for modular multilevel converters when inserting a cold-reserve redundant submodule. IEEE Trans Power Electron 30(8):4052-4057

[30] Konstantinou G, Pou J, Ceballos S et al (2013) Active redundant submodule configuration in modular multilevel converters. IEEE Trans Power Deliv 28(4):2333-2341

Liang ZHANG received the Ph.D. degree from Shanghai Jiao Tong University, Shanghai, China, in 2012. He worked as a postdoc in Tsinghua University, Beijing, China, from 2013 to 2017. He is currently an associated professor in Nanjing Institute of Technology, Nanjing, China. His research interests include interface for renewable energy resources, power quality and high power converters.

Dan ZHANG is currently working on the Ph.D. degree in power electronics and power drives at the Key Laboratory of Control of Power Transmission and Conversion, Shanghai Jiao Tong University, Shanghai, China. His current research interests include multilevel converter and motor drives.

Ting HUA received the Master degree in Nanjing Institute of technology, Nanjing, China in 2018. Her research interest is modular multilevel converter. She now works in State Grid Jiangsu Electric Power Company, Wuxi Power Supply Company, Jiangsu, China.

Jihong ZHU is currently a professor in Tsinghua University, Beijing, China. His research interests include power converters and computer control.

Gang CHEN now works in State Grid Jiangsu Electric Power Company, Jiangsu, China. His research interests include safety quality of power system and power equipment.

Tongzhen WEI received the Ph.D. degree from the Graduate University of Chinese Academy of Sciences, Beijing, China, in 2004. He is currently a professor in the institute of electrical engineering, Chinese Academy of Sciences, Beijing, China. His research interests include flexible AC transmission systems (FACTS), VSC-HVDC and power quality.

Ting YANG received the Master degree from Jiangsu University, Zhenjiang, China, in 2011. She now works in Nanjing Institute of Technology, Nanjing, China. Her research is mainly on power quality and power converters. 